\title{
Pneumocephalus Following Thoracic Surgery with Posterior Chest Wall Resection
}

\author{
Ina Müller ${ }^{1}$ Mario Tönnies ${ }^{1}$ Joachim Pfannschmidt ${ }^{1}$ Dirk Kaiser ${ }^{1}$ \\ ${ }^{1}$ Department of Thoracic Surgery, Helios Klinikum Emil von Behring, \\ Berlin, Germany \\ Thorac Cardiovasc Surg Rep 2015;4:5-7.

\begin{abstract}
Address for correspondence Ina Müller, MD, Department of Thoracic Surgery, Helios Klinikum Emil von Behring, Walterhöferstrasse 11, Berlin 14165, Germany (e-mail: ina.mueller83@hotmail.com; ina. mueller@helios-klinken.de).
\end{abstract}

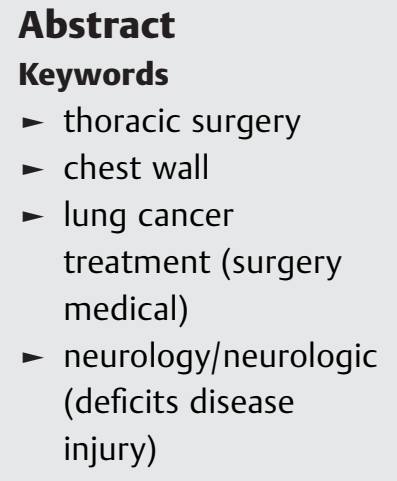

Pneumocephalus can be seen after head injury with fracture of the skull-base or in cerebral neoplasm, infection, or after intracranial or spinal surgery. We report on a 69-year-old male patient with pneumocephalus after right-sided lobectomy and en bloc resection of the chest wall for non-small-cell lung cancer. Postoperatively, the patient showed a reduced vigilance level with no response to pain stimuli and anisocoria. The CCT scan revealed an extensive pneumocephalus; following which, the patient underwent neurosurgery with laminectomy and ligature of the transected nerve roots. After operation the patient returned to his baseline mental status.

\section{Introduction}

Pneumocephalus following thoracic surgery was first reported in 1974 and since then it has been rarely described. Most commonly, pneumocephalus can be seen after head injury with fracture of the skull-base or in cerebral neoplasm, infection, or after intracranial or spinal surgery. We describe a case of pneumocephalus after thoracic surgery with posterior chest wall resection and the necessity of neurosurgical operation.

A 69-year-old patient was admitted to hospital with pain in the right thoracic side and the right shoulder. Computer tomography of the chest showed a T3-tumor in the right upper lobe with local infiltration of the posterior chest wall. A CT-guided biopsy was obtained for histological examination, which showed a poorly differentiated squamous cell lung cancer. The patient received two cycles of neoadjuvant chemotherapy with cisplatin plus vinorelbine and radiotherapy with $45 \mathrm{~Gy}$. Subsequently, the tumor size decreased and the patient underwent surgery ( - Fig. 1). Posterolateral thoracotomy was performed with en bloc resection of the right upper lobe and the involved ribs T2 to T4. Disarticulation of the costotransversal and costovertebral joints were necessary and chest wall reconstruction was done by Gore-Tex (W.L. Gore \& Associates, Inc., Arizona, United States) dual-mesh prosthesis. For pain relief, our patient received a thoracic epidural catheter preoperatively.

On the first postoperative day, the patient presented with a sudden reduced vigilance level, no response to pain stimuli, and anisocoria. CT of the head showed an extensive pneumocephalus of both hemispheres with a collection of free air in the lateral ventricles (-Fig. 2). The epidural catheter was removed at once to exclude one of the possible causes for postoperative pneumocephalus. After two days of conservative therapy with bed rest in strict supine position of the head and body, the patient was conscious without anisocoria. Meanwhile, the patient developed a right-sided pneumonia and consecutive antibiotic therapy was administered. There was a rapid improvement in the patient's condition with the exception of persistent headache and vertigo. Assuming that intracranial hypotension was created by a cerebrospinal fistula, the patient was transferred to the neurosurgical department. In addition, it was assumed that there was an epidural leakage due to the puncture for epidural catheter placement, thus an epidural blood patch by autologous donation was made. However, despite the blood patch, the received

July 27,2014 accepted after revision

October 28, 2014

published online

January 20, 2015
DOI http://dx.doi.org/

10.1055/s-0034-1396683. ISSN 2194-7635. (c) 2015 Georg Thieme Verlag KG
Stuttgart · New York

License terms

((1) $\circledast$ 


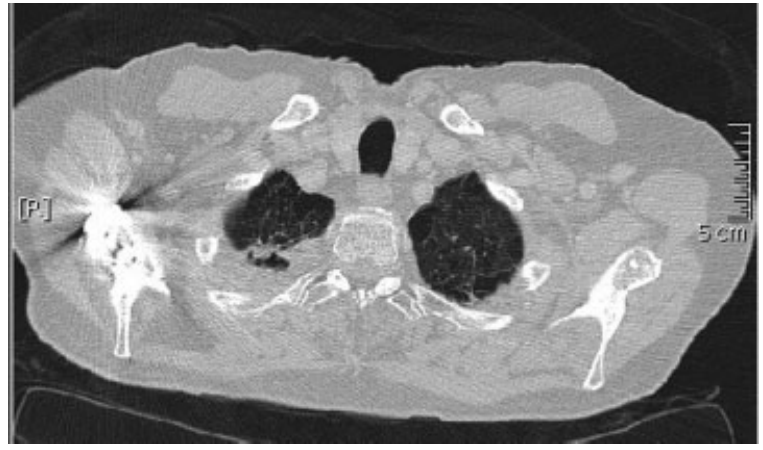

Fig. 1 Computer tomography of the chest after neoadjuvant therapy.

symptoms of vertigo and headache remained. CT scan of the brain showed a complete decline of the pneumocephalus and hyperdensity in the external subarachnoid spaces. Chest X-ray revealed the accumulation of thoracic apical fluid. Under the assumption of a cerebrospinal fluid fistula with the loss of cerebrospinal fluid into the pleural space, a CTguided thoracentesis was performed. Laboratory analysis of the pleural effusion showed high values of $\beta$-trace-protein. Myelography and MRI were carried out for diagnosing and locating of the subarachnoid-pleural fistula, which was detected at the level of the T2 to T3 nerve roots ( $\mathbf{- F i g . ~} \mathbf{3})$. The patient underwent neurosurgery with laminectomy and ligature of the transected nerve roots. After surgery the patient reported a slow decline of headache and vertigo. After 1 month, brain pressure was normalized and the patient did well without neurological symptoms. Two months after the initial thoracic surgical intervention, the patient was discharged home in good general condition.

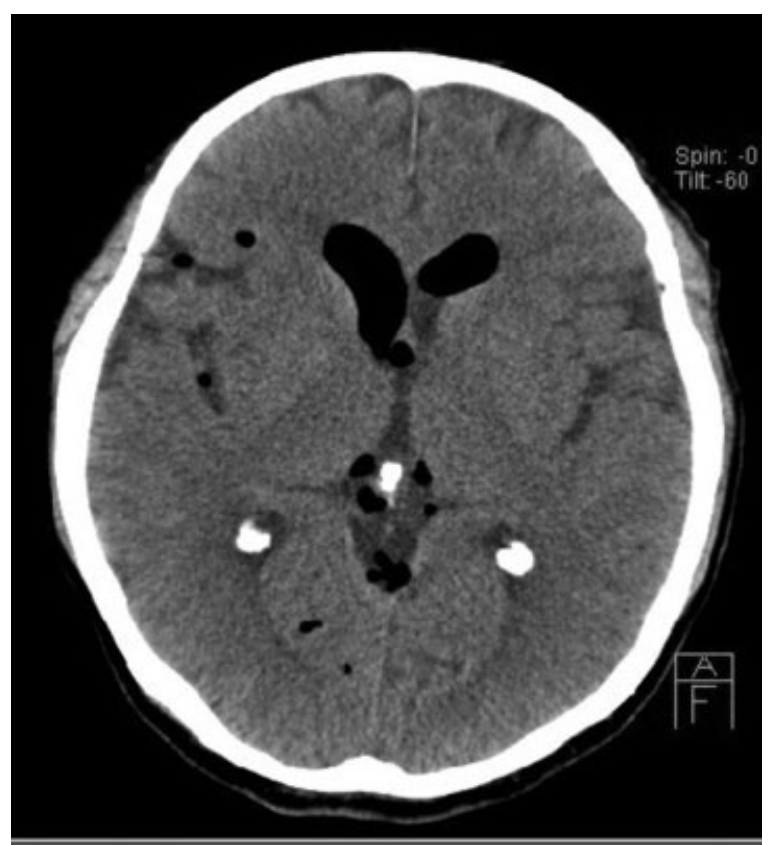

Fig. 2 Pneumocephalus of both hemispheres with a collection of free air in the lateral ventricles.

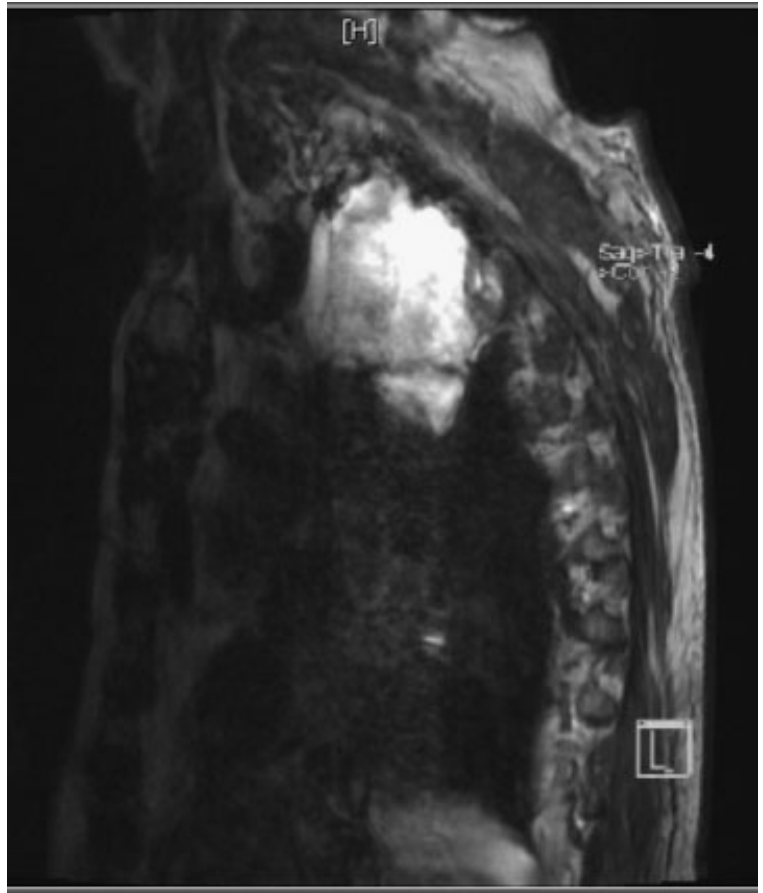

Fig. 3 MRI showed subarachnoid-pleural fistula after chest wall resection.

\section{Comment}

Pneumocephalus is a rare but well-known complication. It can be caused by trauma, neurosurgical procedure, epidural anesthesia, and also as a complication of thoracic surgical procedures. The body of literature is limited to a few articles after thoracic surgery. ${ }^{1,2}$ Symptoms such as headache, vertigo, nausea, vomiting, disorientation, and convulsion are described and may be a complication of meningitis due to pleuro-subarachnoid fistulas. Nevertheless, it was discussed whether pleuro-subarachnoid fistulas are often not recognized in the postoperative course. Moreover, the time till symptoms become evident may vary. In our case, the patient developed first symptoms on the first postoperative day, while other authors describe first symptoms between 1 and 8 wk after operation. ${ }^{1-5}$ Most reported cases of pneumocephalus developed after removal of the chest tube when intrapleural air extends under positive pressure into the subarachnoid space. ${ }^{2}$ Dural tear during rib resections at the costovertebral junction or around the intercostal nerve root facilitates pleuro-subarachnoid fistulas. CT scan of the brain is mandatory to verify the diagnosis. The initial treatment encompasses bed rest, flat-head positioning (Trendelenburg position), and drainage tube placement to control intrathoracic air pressure. An antibiotic prophylaxis against meningitis should be initiated and the patient should be monitored neurosurgically. ${ }^{4,5}$ Complementary or in case of symptom persistence, further investigations should be performed including MRI and myelographic studies to locate the cerebrospinal fistula. ${ }^{1}$ In addition, laboratory tests of the pleural effusion may help to identify a cerebrospinal leakage. Obliteration of a persistent cerebrospinal fistula should be sought 
and this makes laminectomy or a thoracotomy with nerve root ligation usually necessary. After operation, most of the reported patients showed rapid improvement of their neurological symptoms. 1,3

\section{References}

1 Malca SA, Roche PH, Touta A, Pellet W. Pneumocephalus after thoracotomy. Surg Neurol 1995;43(4):398-401

2 de Olaiz Navarro B, León Atance P, Alix Trueba A. [Pneumocephalus and cerebrospinal fluid fistula following removal of a superior sulcus tumor (pancoast tumor)]. Arch Bronconeumol 2004;40(9): 422-425

3 Schuchert MJ, Myers TG, DeGraft-Johnson J, Bejjani GK, Luketich JD, Landreneau RJ. Pneumocephalus after resection of a lung cancer with posterior chest wall involvement. Ann Thorac Surg 2009;87(5):1609-1611

4 Scanagatta P, Leo F, Veronesi G, et al. Pneumocephalus after Pancoast's tumor surgery: to be or not to be conservative? J Cardiovasc Surg (Torino) 2007;48(3):385-387

5 Lucchi M, Dini P, Givigliano F, Ribechini A, Mussi A. Massive pneumoencephalus of late onset after an en bloc resection for lung cancer. J Thorac Cardiovasc Surg 2004;127(6):1836-1838 